\title{
Metabolic plasticity imparts erlotinib- resistance in pancreatic cancer by upregulating glucose-6-phosphate dehydrogenase
}

\author{
Neha Sharma ${ }^{1}$, Alok Bhushan ${ }^{1}$, Jun He ${ }^{2}$, Gagan Kaushal ${ }^{1}$ and Vikas Bhardwaj ${ }^{1 *}$ (D)
}

\begin{abstract}
Pancreatic ductal adenocarcinoma (PDAC) is one of the most malignant forms of cancer. Lack of effective treatment options and drug resistance contributes to the low survival among PDAC patients. In this study, we investigated the metabolic alterations in pancreatic cancer cells that do not respond to the EGFR inhibitor erlotinib. We selected erlotinib-resistant pancreatic cancer cells from MiaPaCa2 and AsPC1 cell lines. Metabolic profiling of erlotinibresistant cells revealed a significant downregulation of glycolytic activity and reduced level of glycolytic metabolites compared to the sensitive cells. The resistant cells displayed elevated expression of the pentose phosphate pathway (PPP) enzymes involved in ROS regulation and nucleotide biosynthesis. The enhanced PPP elevated cellular NADPH/ $\mathrm{NADP}+$ ratio and protected the cells from reactive oxygen species (ROS)-induced damage. Inhibition of PPP using 6-aminonicotinamide (6AN) elevated ROS levels, induced G1 cell cycle arrest, and sensitized resistant cells to erlotinib. Genetic studies identified elevated PPP enzyme glucose-6-phosphate dehydrogenase (G6PD) as an important contributor to erlotinib resistance. Mechanistically, our data showed that upregulation of inhibitor of differentiation (ID1) regulates G6PD expression in resistant cells thus contributing to altered metabolic phenotype and reduced response to erlotinib. Together, our results highlight an underlying role of tumor metabolism in PDAC drug response and identify G6PD as a target to overcome drug resistance.
\end{abstract}

Keywords: Erlotinib resistance, Metabolic reprogramming, Pancreatic cancer

\section{Introduction}

Epidermal growth factor receptor (EGFR), first described in the early 1980s, is a transmembrane tyrosine kinase receptor that is deregulated in various tumors $[1,2]$. Upregulation or mutation of EGFR has been associated with the progression of non-small cell lung carcinoma (NSCLC), pancreatic cancers, colorectal cancers, and glioblastomas, among other tumors. EGFR-targeted therapies, including monoclonal antibodies (e.g., cetuximab)

\footnotetext{
* Correspondence: vikas.bhardwaj@jefferson.edu

'Department of Pharmaceutical Sciences, Jefferson College of Pharmacy, Thomas Jefferson University, Philadelphia, PA, USA

Full list of author information is available at the end of the article
}

and small molecule inhibitors (e.g., erlotinib, gefitinib), have become valuable therapeutic tools. Small molecule inhibitors of EGFR have especially been beneficial for NSCLC and pancreatic ductal adenocarcinoma (PDAC) patients.

PDAC is one of the most malignant forms of cancer with an overall 5 -year survival rate of $8 \%$. With approximately 44,300 deaths in the year 2018, it is the third leading cause of cancer-related deaths in the USA [3]. Late diagnosis, lack of effective treatment options, and drug resistance make PDAC one of the most difficult cancers to treat $[4,5]$. Analyses of PDAC patient samples revealed that EGFR is overexpressed in more than

(c) The Author(s). 2020 Open Access This article is licensed under a Creative Commons Attribution 4.0 International License, which permits use, sharing, adaptation, distribution and reproduction in any medium or format, as long as you give appropriate credit to the original author(s) and the source, provide a link to the Creative Commons licence, and indicate if changes were made. The images or other third party material in this article are included in the article's Creative Commons licence, unless indicated otherwise in a credit line to the material. If material is not included in the article's Creative Commons licence and your intended use is not permitted by statutory regulation or exceeds the permitted use, you will need to obtain permission directly from the copyright holder. To view a copy of this licence, visit http://creativecommons.org/licenses/by/4.0/ The Creative Commons Public Domain Dedication waiver (http://creativecommons.org/publicdomain/zero/1.0/) applies to the data made available in this article, unless otherwise stated in a credit line to the data. 
$40 \%$ of cases and is associated with poor disease prognosis, invasion, and aggressive clinical behavior [6-9]. Inhibition of EGFR in combination with chemo/radiation therapy has been extensively tested in pancreatic cancer patients. In patients with advanced pancreatic cancer, the addition of erlotinib to the treatment regimen improved the overall survival, progression-free survival, and disease control compared to gemcitabine alone [10]. While approximately $53 \%$ of patients in the study displayed positive EGFR expression, the presence of EGFR did not correlate with a favorable drug response [10]. Other studies have demonstrated that the presence of EGFR mutation, development of rash, and the KRAS/ TP53 mutation are predictive biomarkers for erlotinib efficacy in PDAC [11-13]. Despite positive patient response, drug resistance presents significant challenges for the continuous use of erlotinib.

Proteomic analysis of pancreatic cancer found that an altered metabolism consistent with the Warburg effect plays a vital role in PDAC progression [14-16]. Due to these modifications, the cancer cells are dependent on glycolysis for their energy production. The pentose phosphate pathway (PPP) that branches from the glycolysis plays an important role in cellular redox control and nucleotide generation, and is found to be upregulated in various tumors. The altered metabolic profile provides the proliferating cancer cells with rapid ATP synthesis and carbon for the biosynthesis of nucleotides, lipids, and proteins [17]. Recent studies have demonstrated a key role of $c-m y c$ in regulating pancreatic cancer metabolism. The proto-oncogene myc acts downstream of numerous signaling pathways such as PI3K-Akt, MEK-Erk, and Notch and induces a transcriptional response leading to tumorigenesis and cancer progression [18]. In pancreatic cancers, $c-m y c$ regulates global transcription of metabolic genes and induces Kras-mediated metabolic changes [19, 20]. Inhibition of $c$-myc reduced the survival of pancreatic cancers and altered cellular nucleotide pool $[19,21]$. Although a few studies have attempted to evaluate the role of tumor metabolism on cancer chemotherapy resistance, its role in affecting cancer cell response to a targeted therapy such as erlotinib is not well-established [22-24].

Through the study outlined below, we characterized the metabolic alterations that underline erlotinib resistance in PDAC. Further, we investigated the therapeutic potential of targeting the metabolism of tumors that are resistant to anti-EGFR therapy.

\section{Results and discussion}

\section{EGFR tyrosine kinase inhibitor (TKI) resistant cells have} altered metabolic profile

To understand the metabolic regulations in the cells that do not respond to EGFR-targeted therapies, we selected erlotinib-resistant cells by culturing pancreatic cancer cells MiaPaCa2 and AsPC1 in increasing concentrations of erlotinib. Since these cell lines harbor KRAS mutations, p53 mutation, and wild-type EGFR status, they represent a phenotype commonly observed in pancreatic cancers and thus serve as good model systems. The cells were cultured until stable resistant phenotypes Mia$\mathrm{PaCa} /$ Erlo and AsPC/Erlo were achieved. Erlotinibresistance was assessed using MTT assays (Supplemental S1a) and confirmed by clonogenic assay (Fig. 1a and supplemental S1b). Our cell cycle analysis revealed a significant increase in $S$ and $G 2 / M$ phase population in addition to enhanced cellular proliferation (Supplemental S1c and S1d) in resistant cells. The increased proliferation observed in resistant cells was due to increased levels of cyclin D1, cyclin E, and cyclin A as demonstrated by our immunoblot analysis (Supplemental S1e). Although the increased proliferation is not associated with poor therapy response, previous reports have demonstrated that cells with cyclin D1 overexpression respond poorly to EGFR-targeted therapies [25, 26].

Investigation of signaling downstream of EGFR revealed that resistant cells exhibit reduced Erk activation, whereas no apparent effect was observed in the activation of Akt (data not shown). To interrogate the metabolic adaptations, we determined how the central carbon metabolism is altered in the resistant cells. A schematic diagram of the glycolysis channeling metabolites into the tricarboxylic acid cycle (TCA) and pentose phosphate pathway (PPP) is shown in Fig. 1b. Since the upregulation of glycolysis has been associated with reduced tumor response to various therapies, we analyzed the levels of glycolytic enzymes in our resistant cells [27]. Immunoblot and real-time PCR analysis showed that the resistant cells have reduced protein and mRNA levels of glycolytic enzymes (Fig. 1c, d, and Supplemental 2a). We then compared the metabolic phenotype of drugsensitive and drug-resistant cells using Seahorse metabolic analyzer. We found that resistant cells displayed reduced extracellular acidification rate (ECAR, a measure of glycolysis) and elevated oxygen consumption rate (OCR, a measure of oxidative phosphorylation) (Fig. 1e and Supplemental S2b) in comparison to the erlotinibsensitive cells. The results contrast with those of $\mathrm{Ye}$ et al., who reported that an acquired erlotinib-resistant model of lung cancer cells harboring mutant EGFR showed enhanced cellular dependence on glycolysis [28]. Apart from the different cancer types, the cells used in the study also differ in their EGFR status, (they used EGFR mutant cell lines) which may explain the different outcomes. The data suggest that EGFR genetic status plays a critical role in metabolic alterations observed in cancer cells. We then found that the uptake of glucose is significantly reduced in the resistant cells compared to 
a.
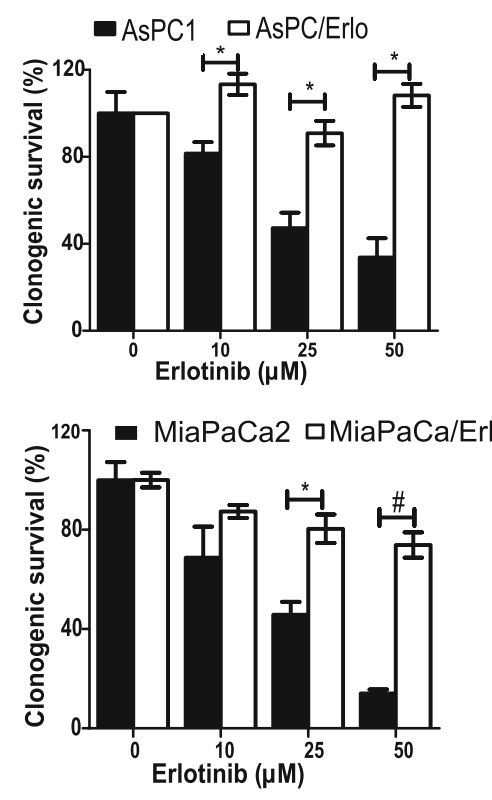

d.

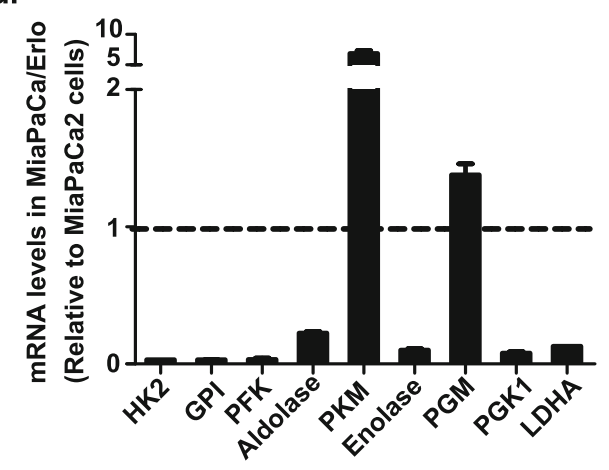

b.

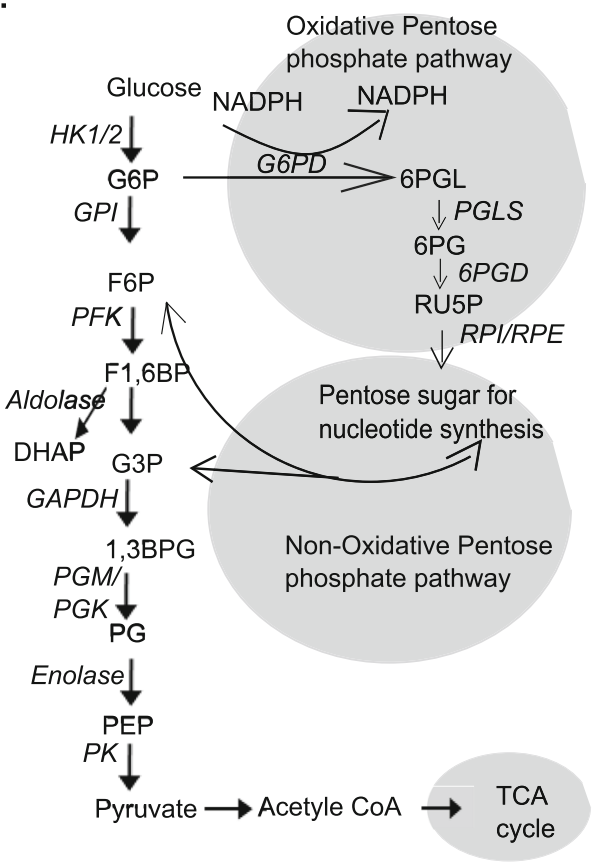

c.

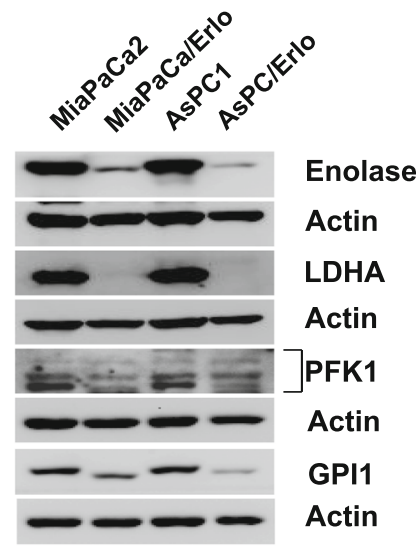

e.

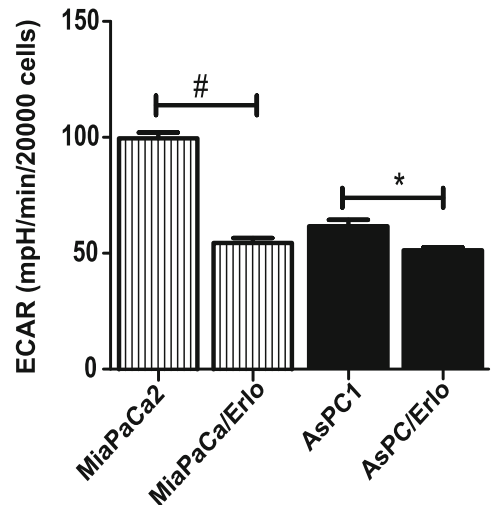

f.

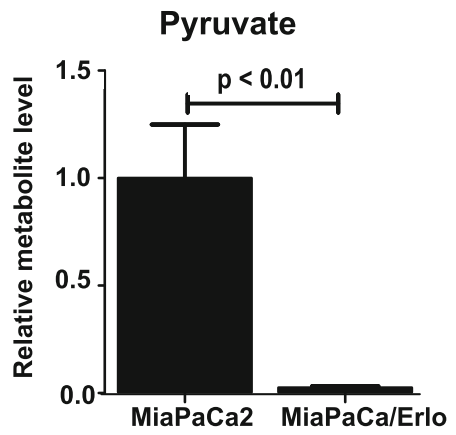

Fig. 1 Erlotinib-resistant cells display downregulated glycolysis. a Effect of erlotinib treatment ( $48 \mathrm{~h}$ ) on the survival of erlotinib-sensitive (MiaPaCa2 and AsPC1) and erlotinib-resistant (MiaPaCa/Erlo and AsPC/Erlo) cells was analyzed using clonogenic assay $(n=3)$. b Representation of central carbon metabolism. $\mathbf{c}$, d Immunoblot and real-time PCR analyses depicting glycolytic enzyme levels in drug-sensitive and drug-resistant cells. HK2, hexokinase 2; GPI, glucose phosphate-isomerase; PFK, phosphofructokinase; PKM, pyruvate kinase M; PGM, phosphoglycerate mutase; PGK, phosphoglycerate kinase; LDHA, lactate dehydrogenase A $(n=2)(n=3)$. e The metabolic phenotype was assessed in cells using Seahorse metabolic analyzer. Graph showing ECAR (glycolysis) levels in sensitive and resistant cells $(n=3)$. $\mathbf{f}$ Pyruvate levels were assessed in cells using LCMS analysis. Graph showing pyruvate levels relative to MiaPaCa2 cells $(n=2)$. Data presented as average \pm SEM $\left({ }^{*} p<0.05,{ }^{\#} p<0.01\right)$

the parent cell line (Supplemental 3a). The low uptake may account for low glycolytic activity in the resistant cells. To confirm the alteration in glycolysis, we performed liquid chromatography-tandem mass spectrometry and found that the resistant cells had lower levels of pyruvate and other key glycolytic metabolites (Fig. If and Supplemental S3b). The results suggest that inhibition of glycolysis may be protective of erlotinib-induced cytotoxicity. To corroborate our findings, we performed clonogenic survival assay in $\mathrm{MiaPaCa} 2$ cells treated with a combination of erlotinib and glycolytic inhibitor, 3bromopyruvate (3BP, an inhibitor of enzyme hexokinase 2 ). Our results show that addition of 3BP induced a non-significant increase in survival of cells compared to cells treated with erlotinib alone (Supplemental S4). Our results show that the downregulation of glycolysis is associated with the poor response of pancreatic cancer cells to erlotinib. 
Erlotinib-resistant cells exhibit upregulated pentose phosphate pathway

To delineate the metabolic deregulation in resistant cells, we performed real-time PCR to analyze changes in enzymes involved in the PPP. We found that the expression of PPP enzymes, ribulose-phosphate 3-epimerase (RPE), and ribulose-phosphate 4-isomerase (RPI) were significantly upregulated in the resistant cells (Fig. 2a and Supplemental S5a). As part of the non-oxidative PPP, RPE and RPI play a crucial role in nucleotide biosynthesis, and their upregulation would support the highly proliferative phenotype of resistant cells (Fig. 1b and Supplemental S1d). Our real-time PCR and immunoblot analyses also showed that the resistant cells had

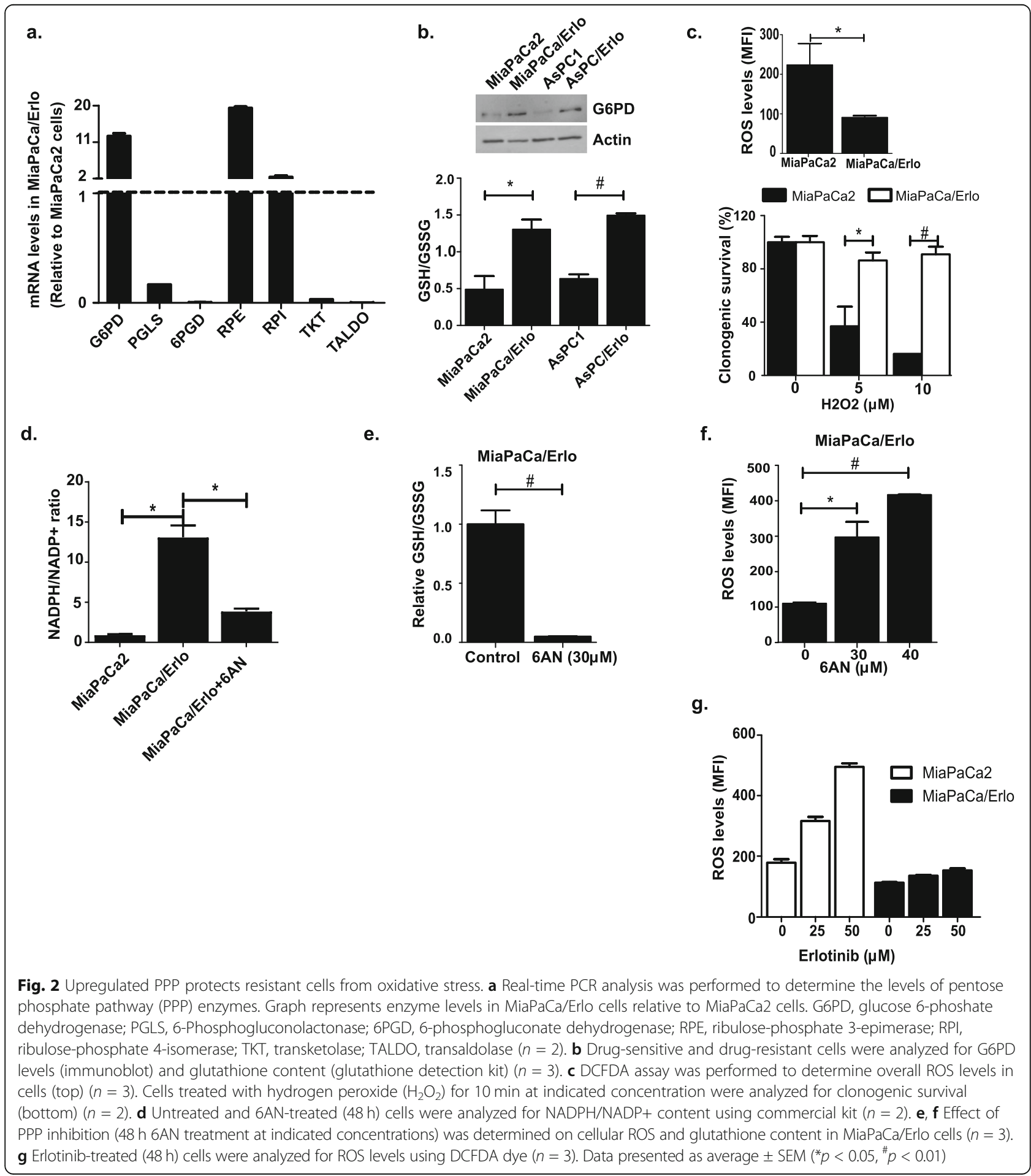


elevated levels of enzyme glucose-6-phosphate dehydrogenase (G6PD) (Fig. 2a, b, and Supplemental S5a). G6PD is the rate-limiting enzyme that channels glycolytic metabolite glucose-6-phosphate into the oxidative PPP. G6PD, along with 6-phosphogluconate dehydrogenase (6PGD), is a key source for reduced nicotinamide adenine dinucleotide phosphate (NADPH) in the cells. The enzyme glutathione reductase utilizes the generated $\mathrm{NADPH}$ to reduce glutathione (GSSG $\rightarrow$ GSH) and therefore maintains the cellular redox balance. However, we found that the level of 6PGD is significantly suppressed in erlotinib-resistant cells. A recent study found that metastatic subclones of pancreatic cancers are dependent on 6PGD for their tumorigenic growth. Interestingly, the authors found that 6PGD derived its substrates from outside of PPP. These substrates (glucuronate and gluconate) successfully elevated the levels of NADPH in metastasized pancreatic cancer cells, however, failed to do so in a non-metastatic pancreatic cell line [29]. In another study, senescence induced by 6PGD knockdown was not associated with inhibition of NADPH levels [30]. This suggests that although both G6PD and 6PGD are part of oxidative PPP, their regulation and metabolical functions are independent of each other.

Consistent with elevated G6PD levels, we found that the resistant cells had an elevated GSH/GSSG ratio compared to the sensitive cells (Fig. 2b). A previous report indicated that an increase in glutathione reduced cellular ROS burden and increased ROS-detoxifying property of cancer cells [31]. Our results showed that both erlotinibresistant cell lines had reduced overall ROS levels compared to the parent cells (Fig. 2c and Supplemental S5b). In addition, the resistant cells were comparatively less sensitive to hydrogen peroxide $\left(\mathrm{H}_{2} \mathrm{O}_{2}\right)$-induced cytotoxicity, indicating that the active antioxidant mechanism protects the cells from oxidative stress (Fig. 2c and Supplemental S5b).

We then confirmed if the altered PPP was responsible for the increased glutathione and lower ROS levels observed in the resistant cells. We found that treatment with oxidative PPP inhibitor 6-aminonicotinamide (6AN) reduced the cellular NADPH/NADP+ ratio and GSH content in the resistant cells leading to elevated ROS levels (Fig. 2d, e, f, Supplemental S5c, S5d, and S5e). Since G6PD is the primary NADPH provider in cancer cells [32], elevated levels of NADPH in cells with suppressed 6PGD could be attributed to enhanced G6PD level and activity. A recent study highlighted that the induction of ROS is essential for cytotoxicity of erlotinib [33]. Hence, we hypothesized that altered metabolic profile would prevent erlotinib-induced ROS generation in resistant cells and thus protect the cells from erlotinib-induced cytotoxicity. We found that treatment with erlotinib $(50 \mu \mathrm{M})$ caused a considerable increase in ROS levels in the sensitive cells (2.7-fold relative to baseline); however, only a small increase in ROS was observed in resistant cells (1.3fold relative to baseline) (Fig. 2g). Our results indicate that upregulation of oxidative PPP protects the resistant cells by regulating the cellular redox capacity through (1) elevated G6PD and glutathione levels and (2) lower ROS levels that protect cells against erlotinib's cytotoxicity.

\section{Inhibition of the oxidative PPP targets erlotinib-resistant cells}

We then hypothesized that the metabolic alterations in resistant cells would change the cell's sensitivity to metabolic inhibitors. To test this, we performed MTT survival assay and found that compared to the sensitive cells, the resistant cells were less responsive to the cytotoxicity of a glycolytic inhibitor iodoacetic acid (IAA, an inhibitor of glycolytic enzyme glyceraldehyde 3phosphate-dehydrogenase) (Supplemental S6a and S6b). Since the resistant cells displayed lower glycolytic activity, their reduced sensitivity to glycolytic inhibitor is on expected lines. The resistant cells were more sensitive to 6-aminonicotinamide-induced cytotoxicity. Interestingly, although erlotinib-resistant cells have upregulated oxygen consumption rate (OCR, Supplemental S2b), their sensitivity to mitochondrial complex inhibitor I, rotenone, was similar to that of erlotinib-sensitive cells (Supplemental S6a and S6b). A possible explanation for this observation is that the oxygen consumption occurs at the later stage (complex IV) of the electron transport chain, whereas, rotenone inhibits the activity of complex I. Hence, similar sensitivity of both erlotinib-sensitive and erlotinib-resistant cells to rotenone may be the result of similar level or activity of electron transport chain complex I in both cell phenotype. Using clonogenic survival assay, we confirmed the enhanced sensitivity of erlotinibresistant cells to 6AN (Fig. 3a). We then investigated whether inhibiting PPP sensitizes the resistant cells to erlotinib. Our results clearly demonstrated that combined treatment of resistant cells with erlotinib and 6AN more effectively reduced the survival of the resistant cells compared to each drug's individual effect (Fig. 3b and Supplemental S7a). Notably, the sensitivity of parent cell lines to erlotinib was not altered by 6AN suggesting that the parent cells are less dependent on oxidative PPP for their survival (Fig. 3b and Supplemental S7a). We then expanded our observations in PANC1 cells that are inherently resistant to erlotinib. We found that treatment with 6AN enhanced the cytotoxicity of erlotinib in PANC1 cells (Supplemental S7b). The results highlight a potentially important role of PPP in cells that are inherently resistant to the drug. Cell cycle analysis revealed that treatment with 6AN leads to the accumulation of cells in the G1 phase and reduced levels of cyclin D1 (Fig. 3c 
a.
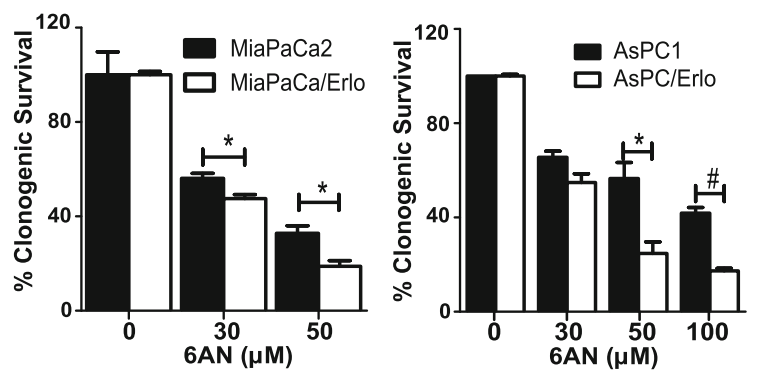

c.

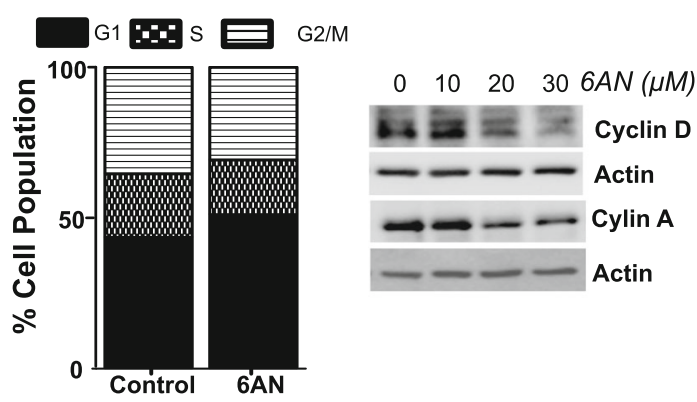

b.
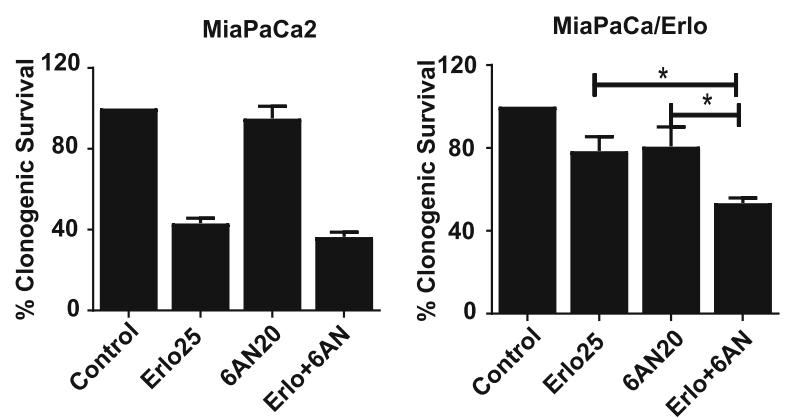

d.

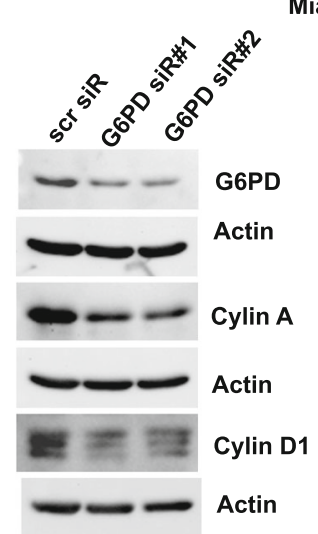

MiaPaCa2/Erlo e.

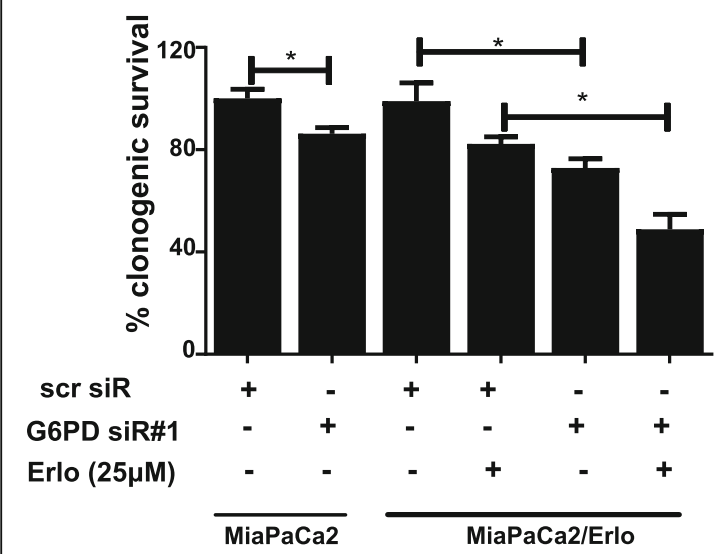

f.

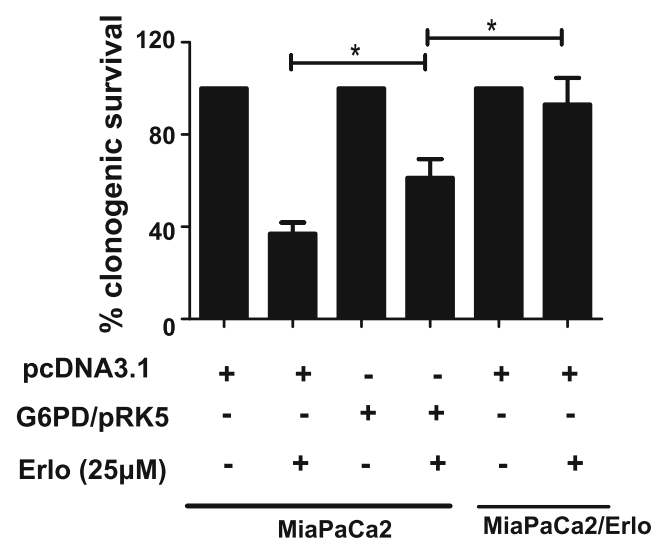

MiaPaCa2 cells

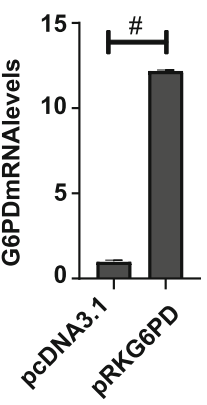

Fig. 3 Resistant cells are sensitive to PPP inhibition. a Graph depicting clonogenic survival of resistant and sensitive cells treated with 6 AN for 48 $\mathrm{h}$ at indicated concentrations $(n=4)$. $\mathbf{b}$ Clonogenic survival assay was performed on drug-sensitive and drug-resistant cells treated with erlotinib in combination with 6AN (48-h combination treatment) $(n=3)$. c Cell cycle analysis was performed on resistant cells treated with $6 \mathrm{AN}(48 \mathrm{~h})$ by flow cytometry using propidium iodide stained cells $(n=4)$. Effect of 6AN on cyclins was ascertained using immunoblot analysis $(n=3)$. d Effect of G6PD knockdown (72-h post siRNA transfection) on MiaPaCa/Erlo cell cycle distribution and cyclin levels was determined $(n=2)$. e Effect of G6PD knockdown on MiaPaCa/Erlo cell sensitivity to erlotinib was determined using clonogenic assay. Comparative effect of G6PD siRNA on survival of MiaPaCa2 and MiaPaCa/Erlo cell was also determined in same experiment $(n=2)$. $\mathbf{f}$ MiaPaCa2 cells transfected with G6PD overexpression plasmid (G6PD/pRK5) were analyzed for their sensitivity to erlotinib using clonogenic survival assay $(n=3)$. The results were compared with empty vector transfected MiaPaCa/Erlo cells that were treated with erlotinib. Data presented as average \pm SEM $\left({ }^{*} p<0.05,{ }^{*} p<0.01\right)$

and supplemental S7c). The observations were consistent with a previous report that showed 6AN halts the cell cycle progression and leads to the accumulation of cells in G1 phase [34]. We then determined the metabolic effect of PPP inhibition. Metabolic analysis found that acute treatment with 
6AN induced a significant increase in the ECAR in erlotinib-resistant cells (1.9-fold), whereas the increase in drug-sensitive cells was less prominent (1.3-fold) (Supplemental S7d). With suppressed glycolysis associated with reduced erlotinib cytotoxicity, upregulation of glycolysis by 6AN treatment may represent a metabolic phenotype that responds well to erlotinib.

Although 6AN targets two PPP enzymes-G6PD and 6PGD, levels of 6-phosphogluconate dehydrogenase were not increased in the resistant cells. Therefore, we hypothesized that the selectivity of 6AN's cytotoxic effect on resistant cells is primarily due to its inhibitory effect on G6PD, and inhibition of G6PD would sensitize the resistant cells to erlotinib. To test our hypothesis, we silenced G6PD using siRNA and found accumulation of cells in G1 phase and downregulation of cyclin D1 and cyclin A levels in erlotinib-resistant cells (Fig. 3d and Supplemental S8a). siRNA-mediated inhibition of G6PD has previously shown to reduce cyclin D1 levels [35]. We then determined how inhibition of G6PD alters the sensitivity of resistant cells to erlotinib. Our results clearly show that G6PD downregulation enhanced erlotinib's cytotoxicity in the resistant cells (Fig. 3e and Supplemental S8b). We also found that inhibition of G6PD reduced the survival of drug-sensitive cells (Fig. 3e). Although the difference in survival of parent and erlotinibresistant cells treated with G6PD siRNA was relatively small, we consistently observed enhanced cytotoxicity of G6PD downregulation on the resistant cells. Our results clearly indicate that the elevated G6PD in erlotinibresistant cells plays a critical role in their survival and blunting the response of erlotinib. The results also highlight that G6PD plays an important role in the survival of erlotinib-sensitive pancreatic cancer cells, which considering the importance of G6PD as an essential NADP $\mathrm{H}$ generator are not surprising.

To further confirm the role of G6PD in promoting erlotinib resistance, we transiently overexpressed G6PD in pancreatic cancer cells and determined their response to erlotinib. Although G6PD overexpression significantly reduced the sensitivity of cells to erlotinib, the extent of protection did not completely recapitulate as observed in the resistant cells (Fig. 3f and Supplemental S8c). This outcome strongly suggests that mechanism alternative to G6PD overexpression could also contribute to erlotinib resistance.

\section{C-myc regulates the metabolic reprogramming of resistant cells}

To delineate the underlying mechanism for metabolic reprogramming, we performed immunoblot analysis and found that resistant cells expressed higher levels of $c$ myc compared to sensitive cells (Fig. 4a). Prior studies have demonstrated that $c-m y c$ plays a central role in tumor cell's metabolic reprogramming [36]. We found that inhibiting c-myc through siRNA suppressed the levels of G6PD in the resistant cells (Fig. 4b). MYC interacts with MAX leading to E-box-dependent transcription of c-myc-induced genes. Using an inhibitor of MYC-MAX interaction, 10058-F4, we found that inhibition of c-myc decreased G6PD levels in the resistant cells (Fig. 4b). The results confirm that c-myc regulates G6PD levels in the resistant cells and plays a central role in upregulation of PPP. To determine the metabolic effect of c-myc inhibition, we performed seahorse analysis and found that acute treatment of c-myc inhibitor enhanced the glycolytic rate (ECAR) in the resistant cells (Fig. 4c). Our results provide key mechanistic insight on how c-myc controls the metabolic alterations in the erlotinib-resistant cells wherein c-myc promotes the oxidative pentose phosphate pathway through upregulation of G6PD. Consistent with our findings, recent reports demonstrated that c-myc plays a role in G6PD transcription through multiple mechanisms $[37,38]$. To uncover the underlying mechanism for c-myc upregulation in resistant cells, we performed immunoblot analysis and found that resistant cells expressed high levels of inhibitor of differentiation 1 (ID1) (Fig. 4d). ID1 is a member of helix-loop-helix (HLH) family of proteins that act as an oncogene. A recent report highlighted that ID1 regulates the levels of c-myc and G6PD in oxaliplatinresistant hepatocellular carcinoma cells, thus activating the PPP [37]. Using ID1-specific siRNA, we found that inhibition of ID1 suppressed the levels of c-myc and G6PD in erlotinib resistant cells (Fig. 4e). Our results suggest that upregulation of ID1 supports the altered phenotype in erlotinib-resistant cells by activating the ID1-c-myc-G6PD axis.

\section{Conclusion}

Overall, our novel findings reveal that pancreatic cancer cells resistant to erlotinib have an altered metabolic profile, and these alterations could potentially be targeted to overcome drug resistance. The altered metabolism is characterized by reduced glycolytic activity in the resistant cells. The upregulation of PPP represents a necessary adaptation that allows the cancer cells to increase their proliferation and keep cellular ROS levels low. The enhanced vulnerability of the resistant cells to metabolic inhibitor such as $6 \mathrm{AN}$ presents an attractive strategy to target cancer cells resistant to erlotinib.

\section{Materials and methods}

\section{Cell culture}

Human pancreatic carcinoma cell lines AsPC1 (CRL1682), MiaPaCa2 (CRL-1420), PANC-1 (CRL-1469), and BxPC-3 (CRL-1687) were obtained from American Type Culture Collection (Manassas VA) and maintained in 


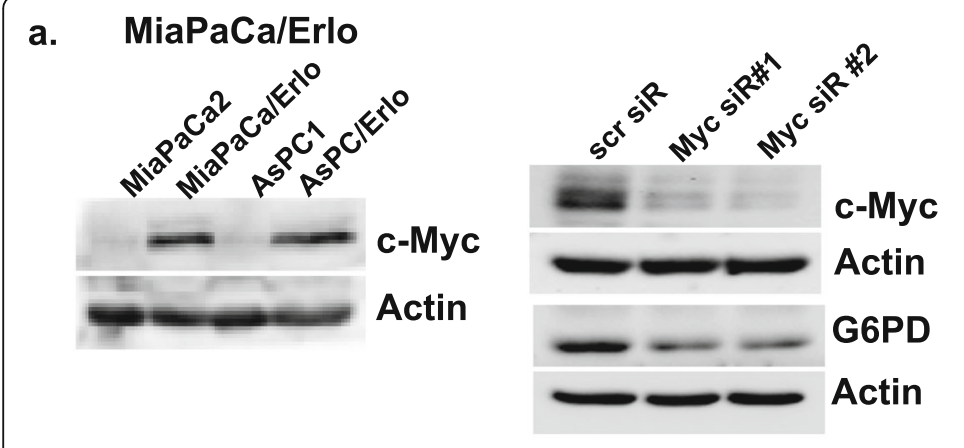

b. MiaPaCa/Erlo

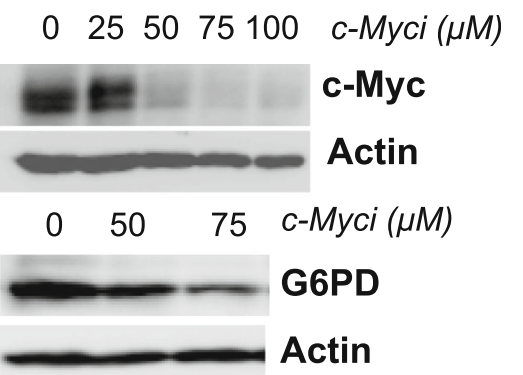

c.

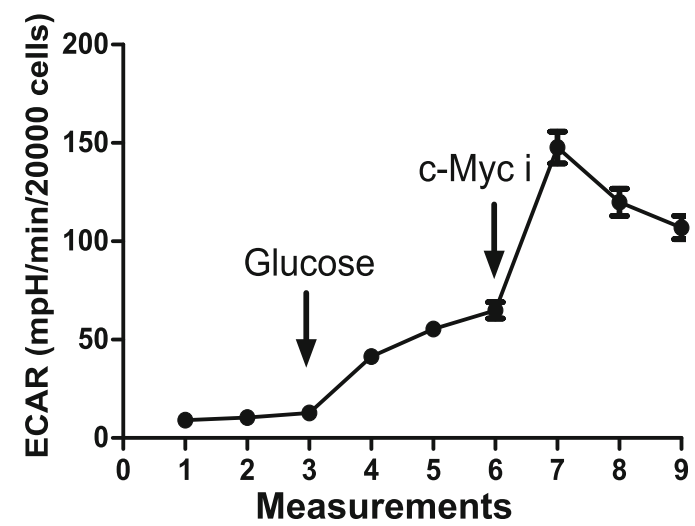

d.

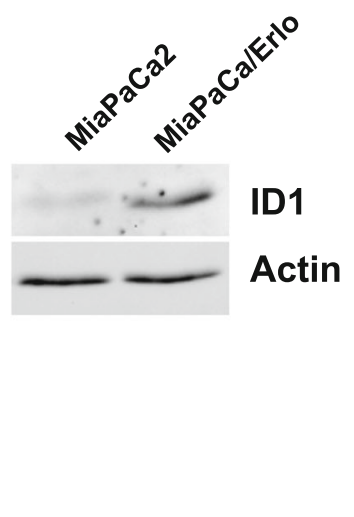

e. MiaPaCa/Erlo

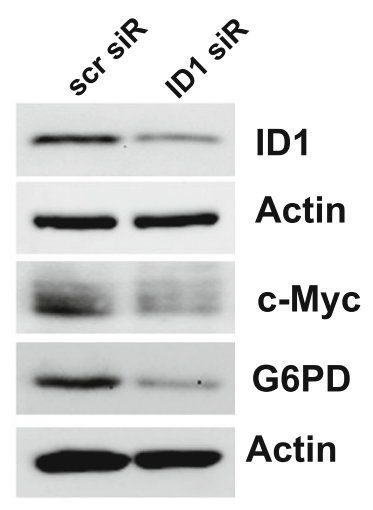

Fig. 4 Myc regulates G6PD levels in resistant cells. a Immunoblot depicting c-myc levels in the resistant cells. Effect of siRNA-mediated downregulation of c-myc on G6PD levels was assessed using immunoblot analysis $(n=3)$. b Effect of c-myc inhibition, c-myci $(10058$ F4, c-myc inhibitor), was analyzed on G6PD levels $(n=3)$. c Effect of acute c-myci treatment on extracellular acidification rate of MiaPaCa/Erlo cells was analyzed using Seahorse metabolic analyzer $(n=2)$. d Levels of ID1 were compared in drug-sensitive and drug-resistant cells using immunoblot analysis $(n=3)$. e Effect of ID1 knockdown was assessed on levels of c-myc and G6PD in MiaPaCa/Erlo cells using immunoblot analysis $(n=3)$

Dulbecco's modified Eagle's medium supplemented with fetal bovine serum and antibiotics in a humidified atmosphere with $5 \% \mathrm{CO}_{2}$ at $37^{\circ} \mathrm{C}$. Cell lines were characterized and authenticated by the supplier.

Generation of stable resistant cell lines (erlotinib-resistant cell lines)

AsPC1 and MiaPaCa2 parental cell lines were treated with increasing concentration of erlotinib $\mathrm{HCl}$ [OSI-744] (Selleckchem, Houston, TX) until stable resistant cell lines were obtained. Cells were cultured in drug-free media for 4-5 days before experiments to prevent acute drug effects.

Drugs, siRNAs, transfection, and plasmid G6PD/pRK5 was a gift from Xiaolu Yang (Addgene plasmid \# 41521). Plasmid transfection was carried out using Lipofectamine 2000 (Invitrogen, Carlsbad, CA). siRNA directed against human c-myc was purchased from Invitrogen (Stealth siRNA) (c-myc siRNA \#1: GCGGAA ACGACGAGAACAGUUGAAA/
UUUCAACUGUUCUGUCGUUUCCGC) and Integrated DNA technology (IDT, Coralville, Iowa) (c-myc siRNA\# 2: CAAACUUGAACAGCUACGGAACUCT, AGAGUU CCGUAGCUGUUCAAGUUUGUG) and transfected using Lipofectamine RNAiMax (Invitrogen). Predesigned siRNAs for G6PD (G6PD siRNA \#1, G6PD siRNA \#2, and ID1 siRNA) were purchased from IDT. G6PD inhibitor 6-aminonicotinamide (6AN) and c-myc inhibitor 10058-F4 were purchased from Selleckchem. 3Bromopyruvate was purchased from Sigma-Aldrich (St. Louis, MO).

\section{MTT survival assay}

MTT survival assay was performed as mentioned previously [39]. Briefly, treated cells were incubated with $20 \mu \mathrm{L}$ of MTT reagent (3-(4, 5-dimethylthiazolyl-2)-2, 5diphenyltetrazolium bromide) made in $1 \mathrm{X}$ PBS at $5 \mathrm{mg} /$ $\mathrm{ml}$ for $4 \mathrm{~h}$, and formazan crystals were dissolved in DMSO to determine cell survival. 


\section{Clonogenic survival assay}

Parental (2000 cells/well) and resistant (1000 cells/well) cells were plated in a 6-well plate and allowed to attach overnight. The cells were then treated with the mentioned drugs for indicated times and allowed to grow drug-free for 7 days. Colonies were stained with 5\% crystal violet stain in $80 \%$ methanol. Image J was used to calculate the staining intensity (ImageJ.nih.gov).

\section{Immunoblot analysis}

Immunoblot assays were performed as mentioned previously [39]. BioRad DC assay was used to determine protein content. $\beta$-Actin was used as the loading control. Information for antibodies can be found in supplementary information SI1.

\section{Cell cycle analysis}

Treated cells were collected and washed with cold 1XPBS. Cells were fixed using ice-cold $80 \%$ ethanol solution overnight at $-20^{\circ} \mathrm{C}$. The cells were then stained with propidium iodide (PI) $(50 \mu \mathrm{g} / \mathrm{ml})$ in the presence of RNase A $(100 \mu \mathrm{g} / \mathrm{ml})$ and analyzed using flow cytometry (Guava EasyCyte, Millipore Sigma, Burlington, MA).

\section{RNA extraction and real-time polymerase chain reaction} Total RNA was extracted using the RNAeasy kit (Qiagen, Germantown, MD) or triazole (Invitrogen) according to the manufacturer's recommendations. RNA content was quantified using nanodrop, and $2 \mu \mathrm{g}$ of RNA was reverse transcribed using the High Capacity cDNA Reverse Transcription Kit (Applied Biosystems, Foster City, CA) as per the manufacturer's protocol. Quantitative RT-PCR was performed using the Quant3 Studio (Invitrogen) using specific mRNA primers (IDT). Primer sequences are available in supplementary information SI1. HPRT (hypoxanthine guanine phosphoribosyl transferase) was used as the reference gene.

\section{Reactive oxygen species assay}

Cellular ROS was determined using 2', 7'-dichlorofluorescin diacetate (DCFDA) (Sigma-Aldrich) according to the manufacturer's protocol. Briefly, cells (treated as indicated) were incubated with $10 \mu \mathrm{M}$ DCFDA dye in serum-free media for $30 \mathrm{~min}$ at $37^{\circ} \mathrm{C}$, washed with PBS, and analyzed using flow cytometry.

\section{Glucose uptake assay}

Cellular glucose uptake was determined using 2-deoxy2-[(7-nitro-2,1,3-benzoxadiazol-4-yl)amino]-D-glucose (2NBDG) dye (Sigma-Aldrich) according to manufacturer's protocol. Briefly, cells were incubated with $20 \mu \mathrm{M}$ 2NBDG in glucose-free media for $30 \mathrm{~min}$ at $37^{\circ} \mathrm{C}$, washed with PBS, and analyzed using flow cytometry.

\section{Seahorse metabolic assay}

Cellular metabolic analyses were performed using Seahorse metabolic analyzer (Agilent, Santa Clara, CA). Cellular phenotypic and glycolysis stress tests were performed according to the manufacturer's recommendations. 15,000-20,000 cells were plated depending on the cell line. Data was normalized to protein content. Acute treatment for G6PD and c-myc inhibitors were carried out using customized protocol within glycolysis stress test according to the manufacturer's recommendations.

\section{GSH/GSSG and NADPH/NADP+ measurement}

Measurement of cellular glutathione content (GSH and GSSG, Glutathione Colorimetric Detection Kit, Thermo Fisher, Waltham, MA) and NADPH/NADP+ levels (NADP/ NADPH Colorimetric kit, Biovision, Milpitas, CA) was performed following the manufacturer's recommendations.

\section{Metabolite analyses using mass spectrometry}

$\mathrm{MiaPaCa} 2$ and $\mathrm{MiaPaCa} /$ Erlo cells cultured in 100-mm dishes were washed twice with $1 \mathrm{X}$ PBS and collected following trypsinization. The metabolites were collected using $80 \%$ cold methanol followed by centrifugation at 14 , $000 \times g$ for $10 \mathrm{~min}$ at $4{ }^{\circ} \mathrm{C}$. The supernatant was collected and dried in a SpeedVac at $30^{\circ} \mathrm{C}$. The extract was dissolved in $200 \mu \mathrm{l}$ of MS-grade water by vortexing on ice for $10 \mathrm{~min}$ and centrifuged at $14,000 \times \mathrm{g}$ for $10 \mathrm{~min}$. The supernatant was then subjected to liquid chromatographytandem mass spectrometry (LC-MS) analyses.

Liquid chromatography (LC) analysis was performed using a Dionex Ultimate 3000 HPLC system (Thermo Fisher) attached with Thermo Orbitrap mass spectrometer using the XBridge ${ }^{\circ}$ Amide $3.5 \mu \mathrm{m}, 4.6 \times 100 \mathrm{~mm}$ (Waters", Milforfd, MA) column. The mobile phase consisted of 10 $\mathrm{mM}$ ammonium acetate buffer ( $\mathrm{pH} 9.3$ ) and acetonitrile (50:50, \%v/v). The injection volume was $5 \mu \mathrm{l}$, with a flow rate of $0.20 \mathrm{ml} / \mathrm{min}$. The column temperature was maintained at $25^{\circ} \mathrm{C}$. The data acquisition from mass spectrometry was delayed for $1 \mathrm{~min}$ followed by $5 \mathrm{~min}$ of data acquisition for a total of 6 min run time. The analysis was performed under the negative ionization monitoring mode with a heated ion transfer capillary. The Exactive (v.1.1SP6) software was used for mass spectrometry method development and data acquisition. Thermo Xcalibur (v. 3.0.63) was used for integration of Chromeleon, and Exactive was used for sample injections and LC-MS data was acquired in .raw file format.

\section{Mass spectrometry conditions}

A scanning mass range of $160-340 \mathrm{~m} / \mathrm{z}$ was used for all samples. All the scans were performed under negative ion mode, and ionization was achieved using electron spray ionization. The sheath gas flow rate was maintained at 20 psi, auxiliary gas flow rate was maintained at 
5 psi, electron spray voltage used for the ionization was kept at $3.5 \mathrm{kV}$, and the capillary temperature was set to $375^{\circ} \mathrm{C}$. Capillary, tube lens, and skimmer voltages were kept at $-47.50 \mathrm{~V},-105.0 \mathrm{~V}$, and $-22 \mathrm{~V}$, respectively. These parameters were selected and saved as direct infusion method for using the Thermo Exactive (v. 1.1 Sp6) software of Thermo Exactive Orbitrap Mass spec. This method was integrated with HPLC to create a single method for LC-MS runs. The metabolite levels were determined by comparing them with reference peaks obtained from metabolite standards (Sigma-Aldrich), and relative peak area was determined.

\section{Statistical analysis}

Student's $t$ test was used to analyze statistical significance between two groups. The difference was considered significant if $p<0.05$. Results are expressed as average \pm SEM if not specifically indicated.

\section{Supplementary information}

Supplementary information accompanies this paper at https://doi.org/10. 1186/s40170-020-00226-5.

Additional file 1. Supplemental S1: (a) MTT analysis were performed to determine the effect of erlotinib on drug-sensitive and resistant cells ( $n=$ 3). (b) Representative clonogenic survival assay images are shown corresponding to Figure 1a. (c) Cell cycle analysis of erlotinib-sensitive and -resistant cells performed on propidium iodide stained cells $(n=2)$. (d) 15 000 cells were plated for indicated cell lines and cellular proliferation was assessed using cell count assay $(n=3)$. (e) Levels of cyclins were determined in sensitive and resistant cells using immunoblot analysis $(n=3)$. Data presented as average \pm SEM (*, $p<0.05, \#, p<0.01)$.

Additional file 2. Supplemental S2: (a) Real-time PCR analysis depicting altered glycolytic enzyme mRNA levels in AsPC/Erlo cells $(n=2)$. (b) Oxygen consumption rate was analyzed by phenotypic assay using Seahorse Metabolic analyzer $(n=3)$. Data presented as average \pm SEM $(\#, p<0.01)$

Additional file 3. Supplemental S3: (a) Cell labeled with 2-NBDG were analyzed for glucose uptake using flow cytometric analysis $(n=4)$. (b) Graph represents glycolytic metabolite levels in MiaPaCa2 and MiaPaCa/ Erlo cells as measured by liquid chromatography-tandem mass spectroscopy. G6P, glucose 6-phosphate; G3P, glyceraldehyde 3-phosphate; 3PG, 3-phosphoglycerate; PEP, phosphoenolpyruvate. Metabolite level is presented as relative to MiaPaCa2 cells $(n=2)$. Data presented as average \pm SEM (*, p < 0.05).

Additional file 4. Supplemental S4: MiaPaCa2 cells treated with indicated concentration of erlotinib (Erlo) and 3-bromopyruvate (3BP) for 48 hours were analyzed for clonogenic survival $(n=2)$.

Additional file 5. Supplemental S5: (a) Graph representing altered pentose phosphate pathway (PPP) enzyme mRNA levels in AsPC/Erlo cells as measured by real-time PCR analysis $(n=3)$. (b) DCFDA stained cells were used to determine ROS levels in the cells (left). Cells treated with hydrogen peroxide $(30 \mu \mathrm{M})$ for 10 minutes were analyzed for clonogenic survival (right) $(n=3)$. (c) Reduced (GSH) and oxidized (GSSG) glutathione levels were analyzed using glutathione assay kit. Graph representing relative GSH/GSSG content in cells treated with 6AN for 48 hours $(n=3)$. (d) NADPH/NADP levels were analyzed in indicated cells using commercial kit $(n=2)$. (e) The effect of $6 A N$ on the induction of ROS was determined using DCFDA stained AsPC/Erlo cells $(n=4)$. Data presented as average \pm SEM (*, $p<0.05, \#, p<0.01)$.

Additional file 6. Supplemental S6: MTT assays were performed to determine the sensitivity of (a) MiaPaCa2 and MiaPaCa/Erlo, and (b) AsPC1 and AsPC/Erlo cells to metabolic inhibitors to pentose phosphate pathway (6AN), oxidative phosphorylation (Rotenone) and glycolysis (lodoacetic acid). Data presented as average $\pm \operatorname{SEM}(n=3)(*, p<0.05$, \#, $\mathrm{p}<0.01)$.

Additional file 7. Supplemental S7: (a) Effect of 6AN on sensitivity of AsPC1 and AsPC/Erlo cells to erlotinib (Erlo) was determined using clonogenic assay $(n=3)$. (b) Graph depicting sensitivity of pancreatic cancer cell lines (PANC-1, MiaPaCa2, AsPC1, and BxPC-3) to erlotinib (72hour treatment) as measured by MTT assay (left). The effect of 6AN (48hour treatment) on cytotoxicity of Erlotinib on PANC-1 cells was measured using clonogenic survival assay $(n=3)$. (c) Effect of 6AN on cell cycle was determined using propidium iodide stained cells (left). Immunoblot analysis were performed to determine alteration in cyclin levels by 48-hour 6AN treatment (right) $(n=4)$. (d) Effect of acute 6AN treatment (30 uM) on extracellular acidification rate of MiaPaCa2 and MiaPaCa/Erlo cells was assessed using Seahorse metabolic analyzer $(n=3)$. Data presented as average \pm SEM (*, $p<0.05, \#, p<0.01)$.

Additional file 8. Supplemental S8: (a) Effect of G6PD knockdown (72 hours post siRNA transfection) on AsPC/Erlo cell cycle distribution was determined using flow cytometry $(n=2)$. (b) Effect of G6PD knockdown on AsPC/Erlo cell sensitivity to erlotinib was determined using clonogenic assay $(n=3)$. (c) AsPC1 cells transfected with G6PD overexpression plasmid (G6PD/pRK5) were analyzed for their sensitivity to erlotinib using clonogenic survival assay. The results were compared with empty vector transfected AsPC/Erlo cells treated with erlotinib $(n=3)$. Data presented as average $\pm \operatorname{SEM}\left(^{*}, p<0.05, \#, p<0.01\right.$.

Additional file 9. Supplementary Information 1.

\section{Abbreviations}

EGFR: Epidermal growth factor receptor; PDAC: Pancreatic ductal adenocarcinoma; PPP: Pentose phosphate pathway; TCA: Tricarboxylic acid cycle; ECAR: Extracellular acidification rate (measure of glycolysis); OCR: Oxygen consumption rate (measure of oxidative phosphorylation); G6PD: Glucose-6-phosphate dehydrogenase; RPE: Ribulose-phosphate 3epimerase; RPI: Ribulose-phosphate 4-isomerase; PGLS: 6-

Phosphogluconolactonase; 6PGD: 6-Phosphogluconate dehydrogenase; TKT: Transketolase; TALDO: Transaldolase; HK2: Hexokinase 2; GPI: Glucose phosphate-isomerase; PFK: Phosphofructokinase; PKM: Pyruvate kinase M; PGM: Phosphoglycerate mutase; PGK: Phosphoglycerate kinase; LDHA: Lactate dehydrogenase A; 6AN: 6-Aminonicotinamide; ROS: Reactive oxygen species; DCFDA: 2', 7'-Dichlorofluorescin diacetate (ROS dye); GSH/ GSSG: Reduced glutathione/oxidized glutathione ratio; RT-PCR: Real-time polymerase chain reaction; LCMS: Liquid chromatography mass spectrometry

\section{Acknowledgements}

We thank Jennifer Wilson (Thomas Jefferson University, Philadelphia, PA) for her support in editing this manuscript.

\section{Authors' contributions}

NS and VB designed the study. NS, GK, and VB performed experiments and analyzed results. $G K, A B$, and $J H$ contributed to the interpretation of results and providing important reagents. All authors read and approved the final version of the manuscript.

\section{Funding}

The work was supported by funds from Jefferson College of Pharmacy.

\section{Availability of data and materials}

The datasets used are available from the corresponding author upon reasonable request.

\section{Ethics approval and consent to participate}

Not applicable

\section{Consent for publication}

Not applicable

Competing interests

The authors have no competing interests to declare. 


\section{Author details}

Department of Pharmaceutical Sciences, Jefferson College of Pharmacy, Thomas Jefferson University, Philadelphia, PA, USA. ${ }^{2}$ Department of Pathology, Anatomy \& Cell Biology, Sidney Kimmel Medical College, Thomas Jefferson University, Philadelphia, USA.

Received: 6 December 2019 Accepted: 6 September 2020

Published online: 21 September 2020

\section{References}

1. Gill GN, Buss JE, Lazar CS, Lifshitz A, Cooper JA. Role of epidermal growth factor-stimulated protein kinase in control of proliferation of A431 cells. J Cell Biochem. 1982;19(3):249-57.

2. Todaro GJ, Fryling C, De Larco JE. Transforming growth factors produced by certain human tumor cells: polypeptides that interact with epidermal growth factor receptors. Proc Natl Acad Sci U S A. 1980;77(9):5258-62.

3. American Cancer Society. Cancer facts \& figures 2018. 2018.

4. Rosenberg L. Pancreatic cancer: a review of emerging therapies. Drugs. 2000;59(5):1071-89.

5. Sheikh R, Walsh N, Clynes M, O'Connor R, McDermott R. Challenges of drug resistance in the management of pancreatic cancer. Expert Rev Anticancer Ther. 2010;10(10):1647-61.

6. Boeck S, Jung A, Laubender RP, Neumann J, Egg R, Goritschan C, et al. EGFR pathway biomarkers in erlotinib-treated patients with advanced pancreatic cancer: translational results from the randomised, crossover phase 3 trial AlO-PK0104. Br J Cancer. 2013:108(2):469-76.

7. Lemoine NR, Hughes CM, Barton CM, Poulsom R, Jeffery RE, Kloppel G, et al. The epidermal growth factor receptor in human pancreatic cancer. J Pathol. 1992; 166(1):7-12.

8. Ueda S, Ogata S, Tsuda H, Kawarabayashi N, Kimura M, Sugiura Y, et al. The correlation between cytoplasmic overexpression of epidermal growth factor receptor and tumor aggressiveness: poor prognosis in patients with pancreatic ductal adenocarcinoma. Pancreas. 2004;29(1):e1-8.

9. Yamanaka Y, Friess H, Kobrin MS, Buchler M, Beger HG, Korc M. Coexpression of epidermal growth factor receptor and ligands in human pancreatic cancer is associated with enhanced tumor aggressiveness. Anticancer Res. 1993:13(3):565-9.

10. Moore MJ, Goldstein D, Hamm J, Figer A, Hecht JR, Gallinger S, et al. Erlotinib plus gemcitabine compared with gemcitabine alone in patients with advanced pancreatic cancer: a phase III trial of the National Cancer Institute of Canada Clinical Trials Group. J Clin Oncol. 2007;25(15):1960-6.

11. Wang JP, Wu CY, Yeh YC, Shyr YM, Wu YY, Kuo CY, et al. Erlotinib is effective in pancreatic cancer with epidermal growth factor receptor mutations: a randomized, open-label, prospective trial. Oncotarget. 2015;6(20):18162-73.

12. Haas M, Siveke JT, Schenk M, Lerch MM, Caca K, Freiberg-Richter J, et al. Efficacy of gemcitabine plus erlotinib in rash-positive patients with metastatic pancreatic cancer selected according to eligibility for FOLFIRINOX: a prospective phase II study of the 'Arbeitsgemeinschaft Internistische Onkologie'. Eur J Cancer. 2018;94:95-103.

13. Blasco MT, Navas C, Martin-Serrano G, Grana-Castro O, Lechuga CG, MartinDiaz $L$, et al. Complete regression of advanced pancreatic ductal adenocarcinomas upon combined inhibition of EGFR and C-RAF. Cancer Cell. 2019:35(4):573-587.e6.

14. Liu M, Quek LE, Sultani G, Turner N. Epithelial-mesenchymal transition induction is associated with augmented glucose uptake and lactate production in pancreatic ductal adenocarcinoma. Cancer Metab. 2016;4 19016-0160-x. eCollection 2016.

15. Penny HL, Sieow JL, Adriani G, Yeap WH, See Chi Ee P, San Luis B, et al. Warburg metabolism in tumor-conditioned macrophages promotes metastasis in human pancreatic ductal adenocarcinoma. Oncoimmunology. 2016;5(8):e1191731.

16. Zhou W, Capello M, Fredolini C, Piemonti L, Liotta LA, Novelli F, et al. Proteomic analysis of pancreatic ductal adenocarcinoma cells reveals metabolic alterations. J Proteome Res. 2011;10(4):1944-52.

17. Liberti MV, Locasale JW. The Warburg effect: how does it benefit cancer cells? Trends Biochem Sci. 2016:41(3):211-8.

18. Hessmann E, Schneider G, Ellenrieder V, Siveke JT. MYC in pancreatic cancer: novel mechanistic insights and their translation into therapeutic strategies. Oncogene. 2016;35(13):1609-18.

19. Hayes TK, Neel NF, Hu C, Gautam P, Chenard M, Long B, et al. Long-term ERK inhibition in KRAS-mutant pancreatic cancer is associated with MYC degradation and senescence-like growth suppression. Cancer Cell. 2016; 29(1):75-89.

20. Murakami S, Nemazanyy I, White SM, Chen H, Nguyen CDK, Graham GT, et al. A Yap-Myc-Sox2-p53 regulatory network dictates metabolic homeostasis and differentiation in Kras-driven pancreatic ductal adenocarcinomas. Dev Cell. 2019:51(1):113-128.e9.

21. Santana-Codina N, Roeth AA, Zhang Y, Yang A, Mashadova O, Asara JM, et al. Oncogenic KRAS supports pancreatic cancer through regulation of nucleotide synthesis. Nat Commun. 2018;9(1):4945 -018-07472-8.

22. Kim DJ, Park YS, Kang MG, You YM, Jung Y, Koo H, et al. Pyruvate kinase isoenzyme M2 is a therapeutic target of gemcitabine-resistant pancreatic cancer cells. Exp Cell Res. 2015;336(1):119-29.

23. Park GB, Chung YH, Kim D. 2-Deoxy-D-glucose suppresses the migration and reverses the drug resistance of colon cancer cells through ADAM expression regulation. Anti-Cancer Drugs. 2017;28(4):410-20.

24. Shukla SK, Purohit V, Mehla K, Gunda V, Chaika NV, Vernucci E, et al. MUC1 and HIF-1alpha signaling crosstalk induces anabolic glucose metabolism to impart gemcitabine resistance to pancreatic cancer. Cancer Cell. 2017;32(3):392.

25. Kobayashi S, Shimamura T, Monti S, Steidl U, Hetherington CJ, Lowell AM, et al. Transcriptional profiling identifies cyclin D1 as a critical downstream effector of mutant epidermal growth factor receptor signaling. Cancer Res. 2006:66(23):11389-98.

26. Kalish LH, Kwong RA, Cole IE, Gallagher RM, Sutherland RL, Musgrove EA. Deregulated cyclin D1 expression is associated with decreased efficacy of the selective epidermal growth factor receptor tyrosine kinase inhibitor gefitinib in head and neck squamous cell carcinoma cell lines. Clin Cancer Res. 2004;10(22):7764-74.

27. Icard P, Shulman S, Farhat D, Steyaert JM, Alifano M, Lincet H. How the Warburg effect supports aggressiveness and drug resistance of cancer cells? Drug Resist Updat. 2018;38:1-11.

28. Ye M, Wang S, Wan T, Jiang R, Qiu Y, Pei L, et al. Combined inhibitions of glycolysis and AKT/autophagy can overcome resistance to EGFR-targeted therapy of lung cancer. J Cancer. 2017:8(18):3774-84.

29. Bechard ME, Word AE, Tran AV, Liu X, Locasale JW, McDonald OG. Pentose conversions support the tumorigenesis of pancreatic cancer distant metastases. Oncogene. 2018;37(38):5248-56.

30. Sukhatme VP, Chan B. Glycolytic cancer cells lacking 6-phosphogluconate dehydrogenase metabolize glucose to induce senescence. FEBS Lett. 2012; 586(16):2389-95.

31. Ju HQ, Lu YX, Wu QN, Liu J, Zeng ZL, Mo HY, et al. Disrupting G6PDmediated Redox homeostasis enhances chemosensitivity in colorectal cancer. Oncogene. 2017:36(45):6282-92.

32. Moreno-Sánchez R, Gallardo-Pérez JC, Rodríguez-Enríquez S, Saavedra E, Marín-Hernández Á. Control of the NADPH supply for oxidative stress handling in cancer cells. Free Radic Biol Med. 2017;112:149-61.

33. Shan F, Shao Z, Jiang S, Cheng Z. Erlotinib induces the human non-smallcell lung cancer cells apoptosis via activating ROS-dependent JNK pathways. Cancer Med. 2016:5(11):3166-75.

34. Massey AJ. Modification of tumour cell metabolism modulates sensitivity to Chk1 inhibitor-induced DNA damage. Sci Rep. 2017:7:40778.

35. Cai T, Kuang Y, Zhang C, Zhang Z, Chen L, Li B, et al. Glucose-6-phosphate dehydrogenase and NADPH oxidase 4 control STAT3 activity in melanoma cells through a pathway involving reactive oxygen species, c-SRC and SHP2. Am J Cancer Res. 2015;5(5):1610-20.

36. Hsieh AL, Walton ZE, Altman BJ, Stine ZE, Dang CV. MYC and metabolism on the path to cancer. Semin Cell Dev Biol. 2015;43:11-21.

37. Yin $X$, Tang $B$, Li JH, Wang $Y$, Zhang $L$, Xie XY, et al. ID1 promotes hepatocellular carcinoma proliferation and confers chemoresistance to oxaliplatin by activating pentose phosphate pathway. J Exp Clin Cancer Res. 2017;36(1):166-017-0637-7

38. Yang $X$, Ye $H$, He M, Zhou X, Sun N, Guo W, et al. LncRNA PDIA3P interacts with c-Myc to regulate cell proliferation via induction of pentose phosphate pathway in multiple myeloma. Biochem Biophys Res Commun. 2018;498(1): 207-13.

39. Bhardwaj V, Tadinada SM, Jain A, Sehdev V, Daniels CK, Lai JC, et al. Biochanin A reduces pancreatic cancer survival and progression. Anticancer Drugs. 2014;25(3):296-302.

\section{Publisher's Note}

Springer Nature remains neutral with regard to jurisdictional claims in published maps and institutional affiliations. 